\title{
Lateral Resolution of Imaging Surface-Analytical Instruments as SIMS, AES and XPS: Application of the BAM-L200 Certi- fied Reference Material and Related ISO Standards
}

\author{
W. E. S. Unger ${ }^{1, *}$, M. Senoner ${ }^{1}$, Th. Wirth ${ }^{1}$, S. Bütefisch ${ }^{2}$ and I. Busch ${ }^{2}$ \\ ${ }^{1}$ Bundesanstalt für Materialforschung und -prüfung (BAM), 12200 Berlin, Germany \\ ${ }^{2}$ Physikalisch-Technische Bundesanstalt (PTB), D-38116 Braunschweig, Germany \\ *wolfgang.unger@bam.de
}

(Received: May 12, 2017; Accepted: October 12, 2017)

\begin{abstract}
The certified reference material BAM-L200 is a nanoscale stripe pattern for length calibration and specification of lateral resolution with stripe widths ranging down to $1 \mathrm{~nm}$. BAM-L200 is prepared from a cross-sectioned epitaxially grown layer stack of $\mathrm{Al}_{\mathrm{x}} \mathrm{Ga}_{1-\mathrm{x}} \mathrm{As}$ and $\mathrm{In}_{\mathrm{x}} \mathrm{Ga}_{1-\mathrm{x}} \mathrm{As}$ on a GaAs substrate. Calibration distances, grating periods and stripe widths have been certified by Transmission Electron Microscopy (TEM) with traceability to the length unit. The combination of gratings, isolated narrow stripes and sharp edges of wide stripes offers a plenty of options for the determination of lateral resolution, sharpness and calibration of length scale at selected settings of imaging surface analytical instruments. BAM-L200 will fully support the implementation of the revised International Standard ISO 18516 which is based on knowledge outlined in the Technical Report ISO/TR 19319:2013. Additionally, the determination of the Field of View (FoV) in the small area modes of X-ray Photoelectron Spectroscopy (XPS) is addressed, too. A test sample is introduced for a measurement of the intensity contribution from outside the nominal Field of View (FoV).
\end{abstract}

\section{State of the art}

The previous state of the art of testing the performance of imaging instruments, e.g., Secondary Ion Mass Spectrometry (SIMS), X-ray Photoelectron Spectroscopy (XPS), Auger Electron Spectroscopy (AES) is described in ISO 18516:2006 [1]. In this standard the grid method, the straight-edge method (also known as knife edge method) and the gold-island method are described. All these methods rely on a line scan (which can be also derived from an image of a straight edge, grid mesh or gold islands) across the boundary between to materials $\mathrm{A}$ and B from which different $D_{\text {x-(100-x) }}$ parameters are calculated as is it is shown in Fig. 1 . The $D_{\text {x-(100-x) }}$ parameters are customarily used to express the lateral resolution. Note that one of the materials A or B can be vacuum. The gold-island method can be also exercised as a line scan over a "small dot", the island, from which the FWHM is taken as measure of lateral resolution.

\section{Problems of the straight edge method}

There are some problems with the straight edge method. The first is that for the determination of the $D_{\text {x-(100-x) }}$ parameter different $x$ values are in use. Larger $x$ values result in a virtually better (i.e. smaller) $D_{\mathrm{x}-(100-\mathrm{x})}$ parameter. Topography effects related to sharp edges, bars of grids with $\mu \mathrm{m}$ scaled curvatures, real shape of $\mathrm{nm}$ scaled gold islands on graphite, etc., may result in edge effects, which will have impact on the resulting $D_{\text {x-(100-x) }}$ parameters, specifically at $\mathrm{nm}$ lateral resolution. Edge effects were discussed in the literature based on Monte Carlo simulation or for submicron particle analysis by the $\mathrm{Au}$ ger microprobe $[2,3]$. Another issue is that a systematic underestimation of $D_{\mathrm{x}-(100-\mathrm{x})}$ occurs when the plateau length $L_{\mathrm{pl}}$ left and right to the (chemical) edge is too short (cf. Fig.2). This can happen when, e.g. nm, scaled gold islands are used for specification of lateral resolution at the nm scale. For AES an impact of backscattering has to be considered, too. 

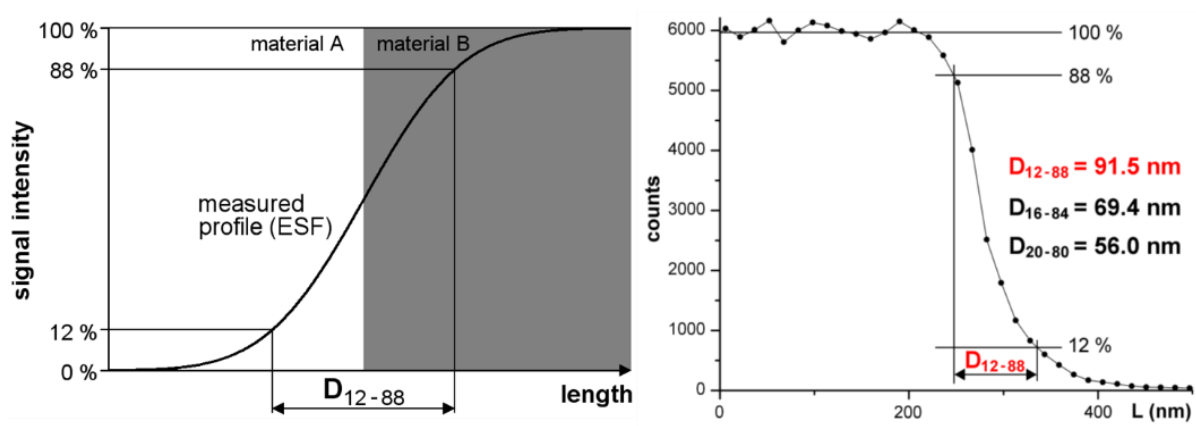

Fig. 1. (color online) Line scan over a straight edge between material A and B, which is representing the Edge Spread Function (ESF). The distance between intensity levels of e.g. $12 \%$ and $88 \%$ of the maximum of a signal characteristic for material A or B is $D_{\text {x-(100-x) }}$ with $\mathrm{x}=12(16$ or 20$)$.

\section{Classical definitions and criteria for lateral resolu- tion of imaging instruments}

Nowadays the performance of imaging SIMS, AES and XPS instruments is substantially improved and approaching the sub $\mu \mathrm{m}$ scale down to less than $10 \mathrm{~nm}$. So it might become questionable whether the straight edge method with the $D_{\mathrm{x}-(100-\mathrm{x})}$ parameter is still appropriate for all relevant cases. Furthermore, it makes sense to consider whether the definition of lateral resolution in surface analysis should not be aligned with definitions used in other, much older communities using imaging instrumentation, too. A classical approach had been introduced by E. Abbe in 1873 when he proposed to use this definition: Lateral resolution of an imaging instrument is the capability for resolution or distinction of microscopic details [5]. Later Lord Rayleigh (1879) defined lateral resolution as follows: Two point sources are just resolved if the central maximum of the intensity diffraction pat- tern produced by one point source coincides with the first zero of the intensity diffraction pattern produced by the other and defined a resolution criterion. This criterion is the decrease of intensity (dip D) by $>19 \%$ between the two maxima. C. M. Sparrow proposed a change of the resolution criterion to $\mathrm{D}>0$ in 1916.

\section{Definitions of lateral resolution in imaging analysis by other ISO TCs}

Other ISO Technical Committees with activity in the field of imaging analysis developed definitions for lateral resolution, too. ISO/TC 202 Microbeam Analysis defines image resolution to be the minimum spacing at which two features of the image can be recognised as distinct and separate [6].

ISO/TC 213 Dimensional and geometrical product specifications and verification defines the "lateral resolution $R_{1}$ as the smallest separation distance between two

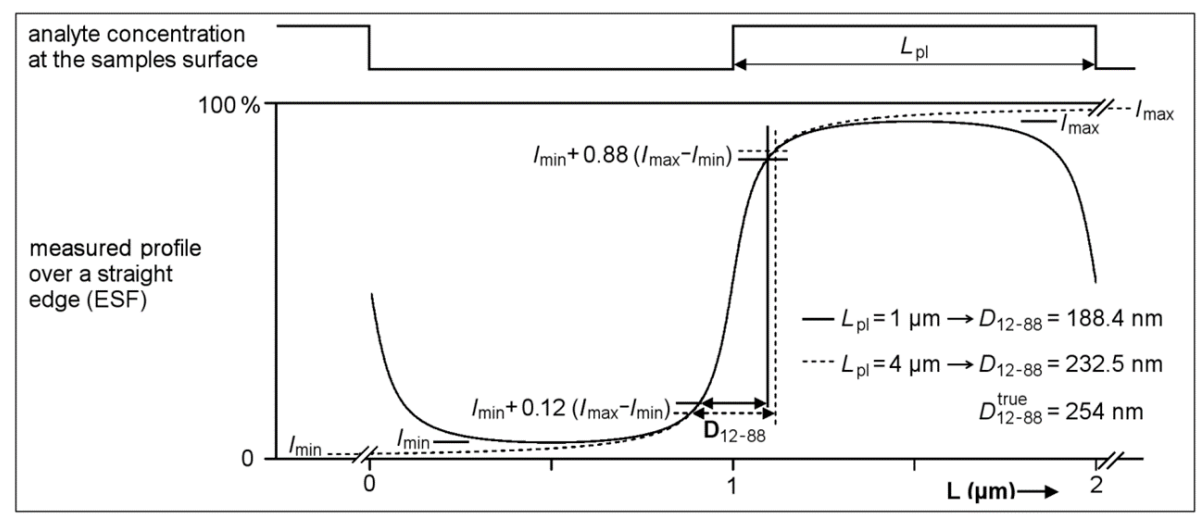

Fig.2. (color online) Simulation of $D_{12-88}$ for two plateau lengths $L_{\mathrm{pl}} 1 \mu \mathrm{m}$ and $4 \mu \mathrm{m}$ and Edge Spread Functions (ESF) which were obtained by convolution of a step transition with a Lorentzian Line Spread Function (LSF) and a FWHM $w_{\text {LSF }}=100 \mathrm{~nm}$. In comparison to the true value of $D_{12-88}$ (which is known because it was used for the simulation and representing the case that $L_{\mathrm{pl}}$ is infinite) the graphically determined value of $D_{12-88}$ is substantially underestimated when $L_{\mathrm{pl}}$ is with $1 \mu \mathrm{m}$ rather short. For $L_{\mathrm{pl}}=4 \mu \mathrm{m}$ the underestimation becomes less than $10 \%$. Ref. [4] delivers a table with recommended $L_{\mathrm{pl}}$ values for specific beam profile parameters (Gaussian and Lorentzian types) for the probe. 


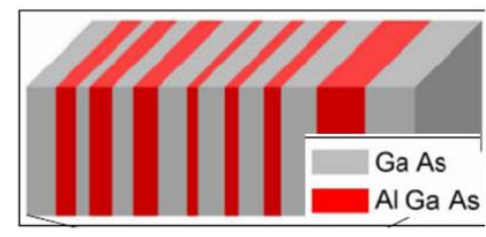

- Cross section of an

AlGaAs - InGaAs - GaAs layer stack

- 142 layers on GaAs substrate

- Thickness of layers between $1 \mathrm{~nm}$ and $700 \mathrm{~nm}$

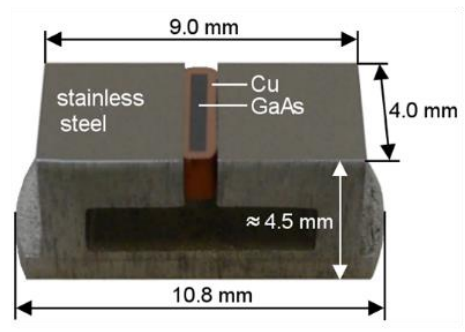

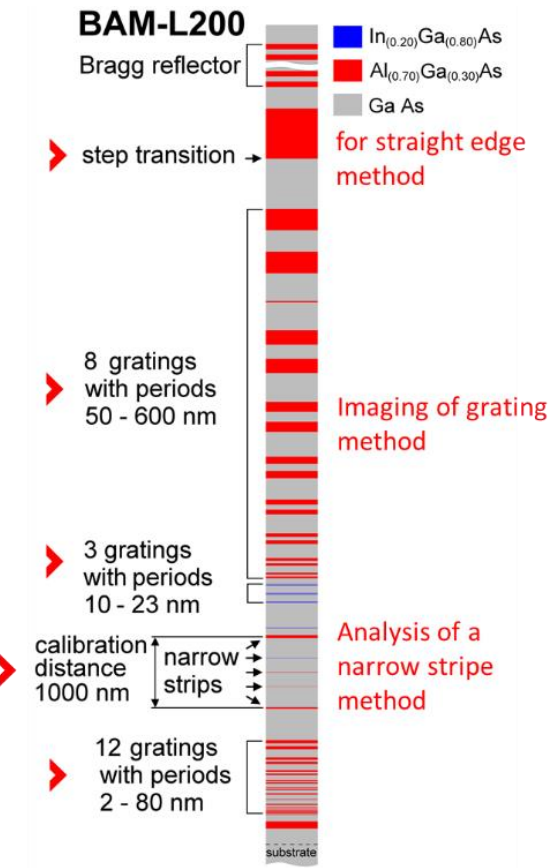

Fig. 3. (color online) Layout and design of the certified reference material BAM L200 for imaging AES and SIMS instruments [8].

features which can be detected" [7].

Both definitions are clearly in line with the classic ones mentioned above in Paragraph 3.

\section{How we can implement the classical definition to imaging surface chemical analysis?}

A definition of lateral resolution in imaging SIMS, AES or XPS surface chemical analysis which is in line with classical ones is: Lateral resolution is the minimum spacing at which two features of the image can be recognized as distinct and separate. To implement this definition some issues have to be addressed:

How to measure it? The answer is to use a test pattern, which delivers graded grating periods as it had been proposed by E. Abbe more than 100 years ago [5]. The test pattern should consist of a set of graded square-wave gratings with low topography. Gratings at low nm dimensions are required for imaging AES and SIMS instruments and on the several $10 \mu \mathrm{m}$ down to high sub $\mu \mathrm{m}$ scale for imaging XPS laboratory instruments.

What about a resolution criterion? A resolved grating should be characterized by a decrease of intensity, i.e. a $\operatorname{dip} D$, between the two imaged grating bars expressed as dip-to-noise ratio $D / \mathrm{s}_{\mathrm{NR}}>4$. For more details see ISO TR 19313-2013 [4].

The final question is whether we have such test sample required to implement the definition above. For imaging
AES and SIMS instruments, the certified reference material BAM L200 (cf. Fig. 3 and ref. [8]) is available in the Web Shop of BAM at www.bam.de. For XPS there are activities at the ETH Zürich (Switzerland) [9] and the German National Metrology Institute, PTB [10], to develop graded gratings having low topography $\mathrm{Au}$ bars embedded in another metal.

Most of the calibration distances, grating periods and stripe widths of BAM L200 have been certified by TEM with traceability to the length unit. The magnification of TEM was calibrated by means of the certified magnification calibration sample (MAG*I*KAL, Norrox Scientific, Canada) which itself is calibrated with respect to the (111) lattice spacing of silicon.

Fig. 4 displays the application of BAM L200 for a determination of the lateral resolution of a ToF SIMS instrument. An image of graded gratings has been taken using $\mathrm{Al}^{+}$secondary ions. In the simplest approach, visual inspection of this image reveals that the lateral resolution must be a little bit better than $31 \mathrm{~nm}$ because this is the smallest period of the grating which is clearly resolved. Following a more sophisticated approach outlined in ref. [4] one may calculate a lateral resolution of $27.2 \mathrm{~nm}$ by interpolation. Principally, as outlined in Ref. [4] the lateral resolution could be also expressed by the FWHM of the line spread function $w_{L S F}$ determined by imaging a narrow line. Fig. 4 shows a result when a 


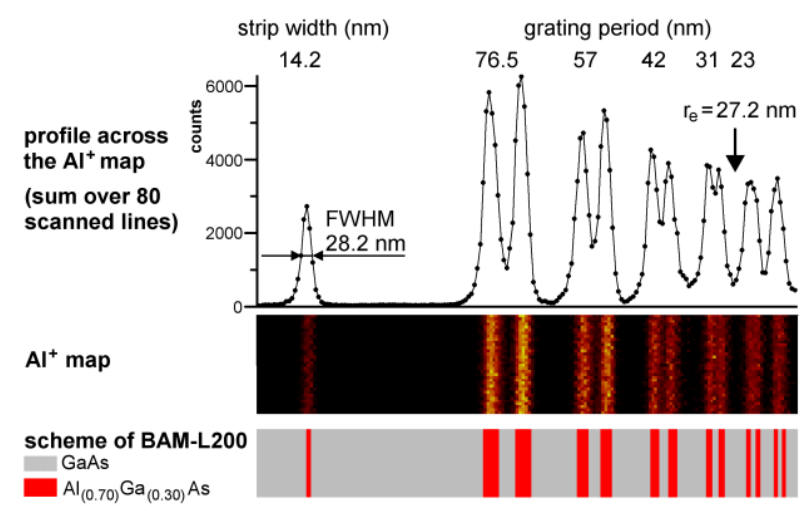

Fig.4. (color online) $\mathrm{Al}^{+}$secondary image of a part of the BAM L200 pattern made of AlGaAs gratings in GaAS. A line scan is prepared from the image data. Gratings with periods from $76.5 \mathrm{~nm}$ down to $31 \mathrm{~nm}$ are resolved. A $14.2 \mathrm{~nm}$ wide AlGaAs stripe was imaged and its FWHM determined, too.

stripe of a width of $14.2 \mathrm{~nm}$ is imaged. However, the $w_{L S F} 28.2 \mathrm{~nm}$ measured in this case is somewhat overestimated because stripe width is a bid too large to be a "narrow line". Fig. 15 of Ref. [4] reveals that for a pure Gaussian LSF the $w_{L S F}$ would be overestimated by $\sim 6 \%$ and for a pure Lorentzian $w_{L S F}$ by $\sim 12 \%$ in the given case. Therefore, for the example presented in Fig. 4 the best estimate for the lateral resolution should be $27 \mathrm{~nm}$ as determined by using the image of graded gratings and the interpolation method introduced in Ref. [4].

\section{Approach and test sample for a determination of the intensity contribution from outside the nominal Field of View of an XPS instrument}

When running an XPS instrument in the small area mode the question arises what an intensity contribution originates from outside the nominal Field of View (FoV) typically specified by the manufacturer measuring the FWHM of the X-ray beam. However, due to the wings of the intensity distribution of the X-ray beam and the instrument's area of acceptance of photoelectrons at the sample there is an intensity contribution from outside this nominal area, which will have impact on a quantitative analysis. For instance, this problem occurs when an analysis of $\mu \mathrm{m}$ scaled solder pads on electronic circuits by quantitative small area/small spot XPS is the task. The impact of signal contributions from outside the pad area has to be minimized by using the right (real) spot size. For a Quantum 2000 X-ray microprobe Scheithauer [11] used Pt apertures with diameters in the range $~ 30$ to $\sim 600 \mu \mathrm{m}$ to measure the intensity contribution from outside the nominal beam diameter. This contribution reaches $50 \%$ for a nominal analysis spot of $15.8 \mathrm{~nm}$ and an aperture diameter of $\sim 30 \mu \mathrm{m}$.

In the SurfChem project the German National Metrology Institute, PTB, developed a prototype test sample for the determination of the FoV in the small area mode of XPS [10]. This sample delivers a sufficient number of $\mathrm{Cr}$ dots and squares on gold in a range of 5 to $200 \mu \mathrm{m}$ diameter and edge length, respectively, on one and the same sample. This design avoids the necessity to have a number of different Pt apertures at hand. Fig. 5 displays a typical result obtained with an XPS instrument in the "small spot" mode. The message of the graph in Fig. 5 is

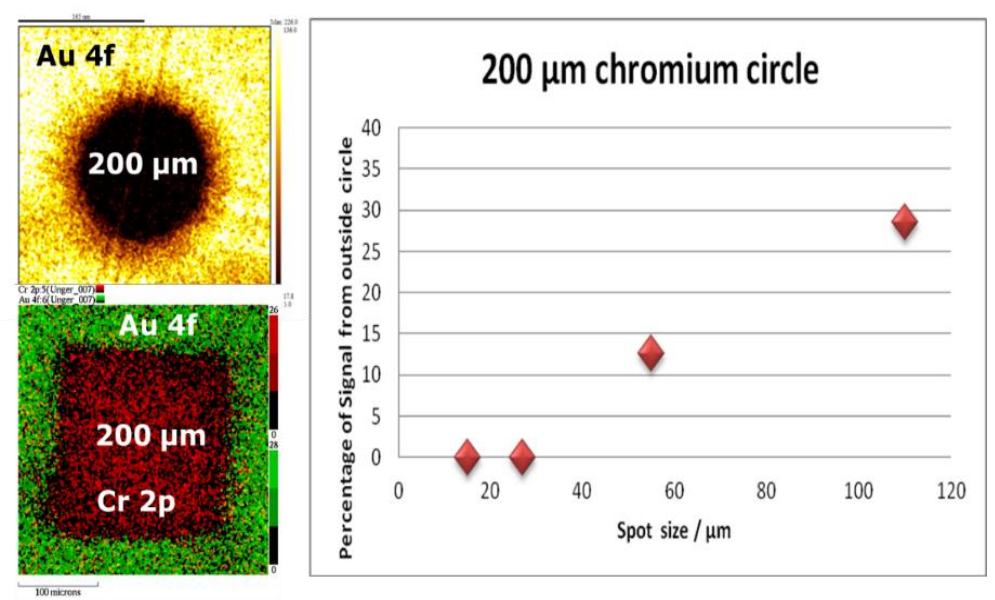

Fig. 5. (color online) Test of PTB's prototype test sample using an AXIS Ultra DLD instrument with a $200 \mu \mathrm{m}$ Cr circle on an Au substrate and nominal FoVs of 15 to $110 \mu \mathrm{m}$ [10]. On the left an Au 4f XPS image for a $200 \mu \mathrm{m}$ circle showing the shadow of the Cr dot and an $\mathrm{Au} \& \mathrm{Cr}$ image overlay of a $200 \mu \mathrm{m} \mathrm{Cr}$ square are shown. Note that the scales are different for both images. The scale bar for the circle is $165 \mu \mathrm{m}$ and that for the square $100 \mu \mathrm{m}$ long. 
that, for example, using a nominal FoV size of $55 \mu \mathrm{m}$ positioned in the center of a $200 \mu \mathrm{m} \mathrm{Cr}$ spot more than $10 \%$ of the measured intensity originates from outside the nominal FoV but for a FoV adjusted to $25 \mu \mathrm{m}$ all the acquired photoelectrons originate from inside the 200 $\mu \mathrm{m}$ Cr spot.

\section{Standardization activities under ISO/TC 201 Sur- face Chemical Analysis}

Comprehensive scientific basics on how to determine lateral resolution for in imaging SIMS, XPS and AES instruments are outlined in ISO/TR 19319:2013. Based thereon a revision ISO 18516 is in preparation by ISO TC/201/SC 2 (recently on DIS stage) which will address straight edge, narrow line and graded gratings based approaches. The new ISO 18516 will expand its scope down to the deep nm scale which is not addressed so far.

The determination and control of the field of view in the small area analysis mode of quantitative XPS is a topic of the VAMAS TWA2 project A22 lead by ETH Zürich and BAM on request of ISO/TC 201/SC 7 „Electron spectroscopies". Based on the results of this VAMAS study the development of a NWIP is expected be launched within the next two years.

\section{Acknowledgement}

This work has been partially funded by the European Metrology Research Program (EMRP) as Project IND15 SurfChem. The EMRP is jointly funded by the EMRP participating countries within EURAMET and the European Union.

\section{References}

[1] ISO 18516:2006, Surface chemical analysis -- Auger electron spectroscopy and X-ray photoelectron spectroscopy -- Determination of lateral resolution, ISO, Geneva, Switzerland, (2006).

[2] Y. G. Li, S. F. Mao, and Z. J. Ding, in Applications of Monte Carlo Method in Science and Engineering, ed. by S. Mordechai, Intech (2011). DOI: $10.5772 / 16171$

[ 3] H. Ito, M.Ito, Y. Magatani, and F. Soeda, Appl. Surf. Sci. 100/101, 152 (1996).

[4] ISO/TR 19319:2013, Surface chemical analysis -Fundamental approaches to determination of lateral resolution and sharpness in beam-based methods,
ISO, Geneva, Switzerland, (2013).

[5] E. Abbe, Beiträge zur Theorie des Mikroskops und der mikroskopischen Wahrnehmung, in: Archiv für mikroskopische Anatomie, Vol. 9, Max Cohen \& Sohn, Bonn, 413 (1873).

[6] ISO 22493:2014, Microbeam analysis -- Scanning electron microscopy - Vocabulary, ISO, Geneva, Switzerland (2014).

[ 7] ISO 25178-601:2010, Geometrical product specifications (GPS) -- Surface texture: Areal -- Part 601: Nominal characteristics of contact (stylus) instruments, ISO, Geneva, Switzerland (2010).

[ 8] M. Senoner, A. Maaßdorf, H. Rooch, W. Österle, M. Malcher, M. Schmidt, F. Kollmer, D. Paul, V.-D. Hodoroaba, S. Rades, and W. E. S. Unger, Analytical and Bioanalytical Chemistry, 407, 3211 (2015). For certificate see: https://rrr.bam.de/RRR/Content /EN/Downloads/RM-certificates/RM-cert-layer-and -surface/bam_1200e.html.

[9] C. Passiu, A. Rossi, L. Bernard, D. Paul, J. Hammond, W. E. S. Unger, N. V. Venkataraman, and N. D. Spencer, Langmuir, 33, 5657 (2017).

[10] EMRP IND 15 SurfChem, Final Report at https://www.euramet.org/research-innovation/emrp/ emrp-calls-and-projects/emrp-call-2010-industry-an d-environment/

[11] U. Scheithauer, Surf. Interface Anal., 40, 706 (2008). 


\section{Discussions and Q\&A with Reviewers}

\section{Reviewer \#1 Mineharu Suzuki (National Institute for Material Science)}

Advantages of the CRM BAM-L200 are introduced with informative descriptions to use practically. This article should be accepted on JSA to realize an evaluation of surface analysis lateral resolutions.

\section{[Q1_1]}

The notation of the parameter $D_{x-(1-x)}$ seems to be confusing. If the unit of $x$ value is percentile, it may be

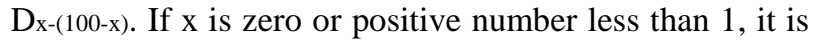
expressed by $\mathrm{D}_{0.2-0.8}$, for instance.

\section{[A1_1]}

We corrected that type by always using $\mathrm{D}_{\mathrm{x}-(100-\mathrm{x})}$.

\section{[Q1_2]}

I recommend the authors for readers to describe how to get (or purchase) the BAM-L200 in this article for readers' convenience.

\section{[A1_2]}

Done.

The certified reference material BAM L200 (cf. Fig. 3 and ref. [8]) is available in the Web Shop of BAM at www.bam.de.

\section{Reviewer \#2 Akira Kurokawa (National Institute of Advanced Industrial Science and technology)}

This paper reports on the lateral resolutions of the surface analysis methods, such as SIMS, AES and XPS, with use of a reference material and ISO standards, which will give the beneficial information to the surface analysis users.

Therefore, I think it is worth to be published from JSA .

\section{[Q2_1]}

Line 4 in Abstract, "Calibration distances, grating periods and stripe widths have been certified by TEM with traceability to the length unit."

TEM calibration procedure does not appear in context, however, the way how TEM measurements assured the SI traceability of the reference material is important information. Please describe it briefly in body text.

\section{[A2_1]}

Details on the calibration procedure are given in a new small paragraph.

\section{[Q2_2]}

Section 2, Line 9, "Effects of field distortions were discussed in the literature....the Auger microprobe."

Is the term of Field Distortion appropriate?

The electric extraction-field near the sample surface does not exist for AES but for SEM.

Ref. 2 uses the term of Edge Effect that reduces the AES spatial resolution.

\section{[A2_2]}

We replaced "field distortion" by "edge effect".

\section{[Q2_3]}

The definition of the true value $D_{12-88}$ is not clearly described. Is it the simulated value supposing that the $\mathrm{L}_{\mathrm{pl}}$ is infinite?

\section{[A2_3]}

The text has been revised accordingly and will be clear now in terms of definition of true value, simulation and infinite $\mathrm{L}_{\mathrm{pl}}$.

\section{[Q2_4]}

Section 5, "Fig.4 shows two lateral resolutions. One is $27.2 \mathrm{~nm}$ derived by using narrow stripes, and the other was $28.2 \mathrm{~nm}$ by a narrow line."

With these two values how do we define the final resolution?

In practical analysis which one is preferable to evaluate a lateral resolution?

\section{[A2_4]}

The text has been fully revised to be clear: Lateral resolution should be measured exploiting the gratings. 